\title{
FIRST RESULTS OF CORRELATION ELECTRON CYCLOTRON EMISSION ON TORE SUPRA
}

\author{
V. S. UDINTSEV* Association EURATOM-Confédération Suisse \\ EPFL/SB/CRPP, Station 13, CH-1015 Lausanne, Switzerland \\ M. GONICHE, J. L. SÉGUI, G. GIRUZZI, D. MOLINA, F. TURCO, G. T. A. HUYSMANS, P. MAGET, \\ and TORE SUPRA TEAM Association EURATOM-Commissariat à l'Energie Atomique \\ CEA/DSM/DRFC, CEA/Cadarache, F-13108 St. Paul-lez-Durance, France
}

\begin{abstract}
A. KRÄMER-FLECKEN Association EURATOM-Institut für Plasmaphysik
Forschungszentrum Jülich GmbH, D-52425 Jülich, Germany
\end{abstract}

Received January 26, 2006

Accepted for Publication May 3, 2006

\begin{abstract}
Measurements of electron temperature fluctuations by means of correlation electron cyclotron emission (ECE) diagnostics aid in understanding the nature of the turbulent transport in fusion plasmas. On Tore Supra tokamak, a 32-channel heterodyne ECE radiometer has been upgraded to include two channels for temperature fluctuation measurements. The central frequency of the yttrium iron garnet filter on each channel is remotely monitored by a driver, allowing one to shift the observation volume in the plasma radially. To resolve the fluctuation amplitude of $0.1 \%$, integration times of $\sim 3 \mathrm{~s}$ at $1 \mathrm{MHz}$ sampling rate are required for the statistical error level of $0.05 \%$. Together with the 32-channel profile radiometer,
\end{abstract}

correlation ECE is a powerful and unique tool for simultaneous observation of the magnetohydrodynamic phenomena and studies of the plasma transport properties in various heating regimes during long-shot multimegawatt operation. First measurements of temperature fluctuations, of various origins, on Tore Supra (including the observation of the toroidicity-induced Alfvén eigenmodes), as well as a description of the analysis methods used in the data evaluation, are reported in this paper.

KEYWORDS: Tore Supra, correlation ECE, temperature fluctuations

\section{INTRODUCTION}

Studies of plasma turbulence are useful for understanding the nature of the transport properties in fusion plasmas. Two general types of fluctuations, electrostatic and magnetic, can be distinguished in tokamak plasmas. Magnetic fluctuations break the nested flux surfaces and, therefore, enhance the plasma transport. Electrostatic fluctuations do not destroy the nested magnetic topology; however, the enhancement of transport is due to the $\vec{E} \times \vec{B}$ drifts from the fluctuating electric fields. ${ }^{1}$ To establish a relation between the fluctuations and transport, measurements of various fluctuating components, such as $\tilde{n}_{e}, \widetilde{T}_{e}, \tilde{B}_{r}$, and $\widetilde{E}_{\theta}$, as well as knowledge of correlations between them, are needed.

Measurements of electron temperature fluctuations $\left(\widetilde{T}_{e}\right)$ by means of correlation electron cyclotron emis-

*E-mail: victor.udintsev@epfl.ch sion (ECE) diagnostics have yielded some interesting information on microturbulence properties both of electrostatic and magnetic origins on TEXT-U (Refs. 2 and 3), W7-AS (Refs. 4 through 6), RTP (Ref. 7), TEXTOR (Ref. 8), Alcator C-Mod (Ref. 9), and some other machines. ${ }^{10}$ These measurements generally require long integration times. Therefore, they will find their best application in experiments in which long (lasting several seconds) stationary plasmas are attained. This is the case on the Tore Supra tokamak $\left(R_{0}=2.40 \mathrm{~m}\right.$, $a=0.72 \mathrm{~m}, B_{T} \leq 4 \mathrm{~T}$, circular cross section), which routinely runs discharges in the range of 10 to $30 \mathrm{~s}$ and which has recently obtained discharges in excess of $6 \mathrm{~min}$ (Ref. 11). The availability of multimegawatt radio frequency (rf) heating [ion cyclotron resonance heating (ICRH), electron cyclotron resonance heating (ECRH), and lower hybrid (LH)] and a possibility to vary the injected power during the shot make Tore Supra a unique machine to study magnetohydrodynamic (MHD) 
phenomena, the plasma transport properties, and their dynamics. Therefore, a 32-channel heterodyne ECE radiometer has recently been upgraded to include two channels for temperature fluctuation measurements with radial and poloidal resolutions of about 1 and $3 \mathrm{~cm}$, respectively. The first series of experiments was performed during the 2004 and 2005 campaigns. The experimental setup and the scientific results, including the first-ever observation of the toroidicity-induced Alfvén eigenmodes (TAEs) by means of ECE on Tore Supra, are presented in this paper. In Sec. II an introduction to the correlation ECE technique that is necessary to understand the analysis made for Tore Supra data is presented. In Sec. III an experimental description of the correlation ECE setup on Tore Supra is given. Estimation of the measurement error is treated in Sec. IV. The first scientific results are shown in Sec. V. Finally, conclusions are drawn in Sec. VI.

\section{CORRELATION ECE TECHNIQUE USED FOR THE ANALYSIS}

In many correlation ECE experiments, ${ }^{2-8}$ the following simplified analysis of data has been used to retrieve the information on temperature fluctuations. The ECE signal $S_{\mathrm{ECE}}(t)$ from the plasma sample volume can be written in a simple form as $^{2}$ :

$$
\begin{aligned}
S_{\mathrm{ECE}}(t) & =\bar{S}_{\mathrm{ECE}}+\tilde{S}_{\mathrm{ECE}}(t)=c\left[\bar{T}_{e}+\widetilde{T}_{e}(t)\right][1+\widetilde{N}(t)] \\
& \approx c \bar{T}_{e}\left[1+\frac{\widetilde{T}_{e}(t)}{\bar{T}_{e}}+\frac{\widetilde{N}(t)}{\bar{T}_{e}}\right]
\end{aligned}
$$

where $c$ is a proportionality (or a calibration) factor. From Eq. (1), an expression for the normalized fluctuation component can be written as follows:

$$
\frac{\tilde{S}_{\mathrm{ECE}}(t)}{\bar{S}_{\mathrm{ECE}}}=\frac{S_{\mathrm{ECE}}(t)-c \bar{T}_{e}}{c \bar{T}_{e}} \approx \frac{\widetilde{T}_{e}(t)}{\bar{T}_{e}}+\frac{\tilde{N}(t)}{\bar{T}_{e}} .
$$

The thermal (or radiation wave) noise $\tilde{N}$ is due to the statistical properties of photons that are measured by the detector and is independent of the plasma electron temperature fluctuations $\widetilde{T}_{e}$. This thermal noise is related to the portion of the frequency spectrum that is collected by the detector, characterized by the $3 \mathrm{~dB}$ intermediate frequency (if) passing bandwidth $B_{\text {if }}$, and to the postdetection video bandwidth $B_{V}$. To have a reasonably good radial resolution, $B_{\text {if }}$ cannot be too large, and $B_{V}$ has to be large enough to allow for fluctuation measurements. Consequently, for relevant values of $B_{V}$ and $B_{\text {if }}, \tilde{N}$ is typically in the range of 3 to $10 \%$, above the temperature fluctuation level in the plasma core that is typically 1 to $3 \%$. The issue of the proper estimation of the noise level in Tore Supra experiments will be treated in detail in Sec. IV. Temperature fluctuation measurement by ECE can there-

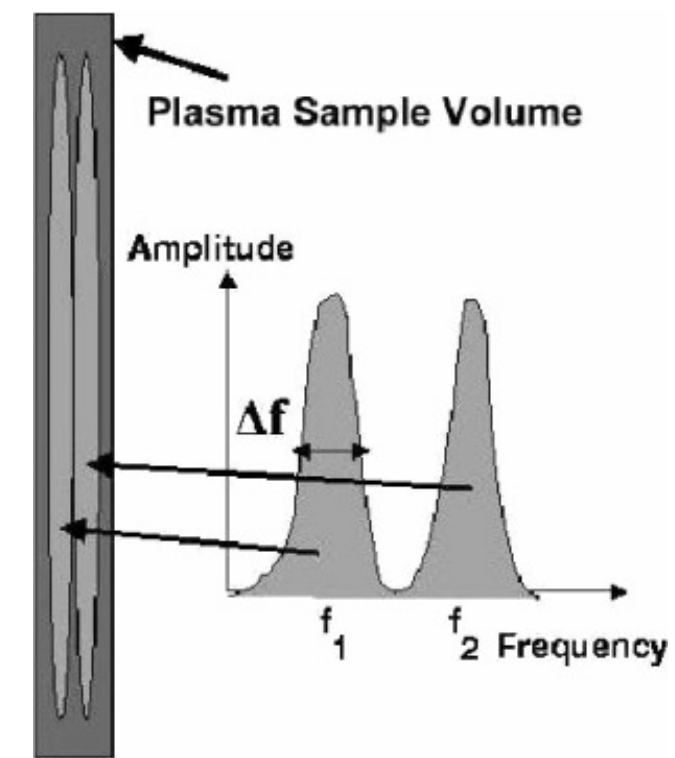

Fig. 1. Thermal noise decorrelation with a single line-of-sight ECE system. Due to the finite line width of ECE radiation, two channels with closely spaced, nonoverlapped frequency bands centered at $f_{1}$ and $f_{2}$ correspond to measurements from almost the same plasma sample volume. $^{15}$

fore be achieved only by cross-correlation between two ECE signals, for which temperature fluctuations are correlated while the noise is uncorrelated. ${ }^{2-6}$ In case of narrowband fluctuations (such as MHD modes, etc.), the situation is even more favorable for the cross-correlation analysis (see Secs. IV.A. and V.B for details).

The scheme in which two sample volumes are separate in frequency space but overlap in physical space allows decorrelation of the thermal noise as long as the fluctuation correlation length is longer than the sample volume spacing (Fig. 1). Another useful application of the correlation ECE is its ability to obtain the dispersion relation when fluctuations propagate in the plasma. ${ }^{12}$

In general, the cross-correlation function over time period $P$ for two normalized fluctuating signals $\left(\tilde{S}_{1}, \tilde{S}_{2}\right)$, and for a given time lag $\tau$, can be written as ${ }^{13}$ :

$$
R_{12}(\tau)=\frac{1}{\bar{S}_{1} \bar{S}_{2} P} \int_{0}^{P} \tilde{S}_{1}(t) \tilde{S}_{2}(t+\tau) d t .
$$

For $\tau=0$ (zero time lag), Eq. (3) takes the following form:

$$
\begin{aligned}
R_{12}(0)= & \overline{\tilde{S}_{1}(t) \tilde{S}_{2}(t)}=\frac{\overline{\widetilde{T}_{e 1}(t) \widetilde{T}_{e 2}(t)}}{\bar{T}_{e 1} \bar{T}_{e 2}}+\frac{\overline{\widetilde{N}_{1}(t) \tilde{N}_{2}(t)}}{\bar{T}_{e 1} \bar{T}_{e 2}} \\
& +\frac{\overline{\widetilde{T}_{e 1}(t) \tilde{N}_{2}(t)}}{\bar{T}_{e 1} \bar{T}_{e 2}}+\frac{\overline{\widetilde{T}_{e 2}(t) \tilde{N}_{1}(t)}}{\bar{T}_{e 1} \bar{T}_{e 2}} .
\end{aligned}
$$


The particular case $\tilde{S}_{1}(t)=\tilde{S}_{2}(t)=\tilde{S}(t)$ defines the autocorrelation function. Whenever both signals are coming from the same plasma volume, one can assume that $\widetilde{T}_{e 1} \approx$ $\widetilde{T}_{e 2}=\widetilde{T}_{e}$. Because the thermal noise is not correlated, the last three terms in Eq. (4) can be neglected, and an expression for the root-mean-square (rms) value of the normalized temperature fluctuations can be obtained:

$$
\frac{\sqrt{\overline{\widetilde{T}_{e}^{2}(t)}}}{\bar{T}_{e}}=\sqrt{R_{12}(0)} .
$$

The cross-correlation method does not require ECE signals to be absolutely calibrated. In many cases, besides the rms estimation of the fluctuations amplitude, it is useful to calculate the cross-spectral density spectrum for two discrete signals, which is the inverse Fourier transform of the cross-correlation function. The onesided cross-spectral density (CSD) for two ECE signals can be defined as follows:

$$
\operatorname{CSD}_{12}=\left|\operatorname{CSD}_{12}(2 \pi f)\right| e^{-j \varphi(2 \pi f)},
$$

where $\left|\operatorname{CSD}_{12}(2 \pi f)\right|$ is the amplitude of the crossspectral density and $\varphi(2 \pi f)$ is the cross phase between two signals.

If broadband modes with a bandwidth $B_{B B}$ exist in the plasma, they cause a peak in the cross-correlation function that decays according the following time scale ${ }^{10}$ :

$$
\tau_{\text {decay }}=\frac{1}{B_{B B}} \sqrt{\frac{\ln 2}{\pi}} \approx \frac{1}{2 B_{B B}} .
$$

A mode with a narrow bandwidth exhibits itself in the cross-correlation function as an envelope of oscillations that decays with increased time lag. In this case, $B_{B B}$ should be replaced with the width of the envelope. A typical example of such mode with a narrow bandwidth will be given in Sec. V.

In estimates of the cross-spectral density, one should take into account that the ECE fluctuation diagnostic has a finite sample volume size. The signals obtained are the averages over the sample volumes. The averaging process attenuates fluctuations with shorter wavelengths, thus limiting the sensitive $k$ (wave number) range of the diagnostic and causing distortion of the measured spectrum and dispersion of the fluctuations. ${ }^{14}$ Usually, ECE radiometers have sample volumes within which the sensitivity is a Gaussian function of space. Following Refs. 14 and 15 , the measured power spectrum of the relative temperature fluctuation $\left|\widetilde{T}_{e} / T_{e}\right|^{2}$ is

$$
S_{m}(k, \Omega)=e^{-(k \cdot d)^{2} / 2} S(k, \omega),
$$

where $k$ is the wave number of the turbulence and $d$ is a characteristic size of the measurement volume. It is seen from Eq. (8) that, due to the finite sample volume sizes, the measured power spectral density will be attenuated by a factor of $\exp \left\{-(k \cdot d)^{2} / 2\right\}$. In tokamaks, it is convenient to define the geometry using toroidal $(\varphi)$ and poloidal $(\theta)$ directions. Fluctuations are homogeneous in the toroidal direction $\left(k_{\varphi} \approx 0\right)$ and thus one can neglect this contribution. The characteristic size of the measurement volume then determines the useful range of $k_{\theta}$ that can be measured. For this experiment, the Millitech Gaussian Optics Lens Antenna (GOLA) located in the equatorial midplane at the low field side (LFS) is used in the front end of the radiometer. The unfocused quasi-parallel Gaussian beam has a poloidal $1 / e$ folding radius $d_{\theta}=$ $3 \mathrm{~cm}$ (the full beam waist in the poloidal direction is $w=2 d_{\theta}$ ) near the plasma center. This value is corrected for diffraction effects. The radial width of the sample volume (i.e., along the direction of beam propagation) is determined by the bandwidth of the intermediate frequency filters and by the relativistic broadening of the ECE, which depends mostly on the electron temperature (see Sec. III and Ref. 16 for details). The radial 1/e folding length $d_{r}$ varies between 0.4 and $1.7 \mathrm{~cm}$ for the reported experiments. From Eq. (8), the minimum values of $k_{\theta, r}$ to which the diagnostic is dominantly sensitive can be defined as values for which the attenuation of the signal is $1 / e$ :

$$
\left(k_{\theta, r} d_{\theta, r}\right)^{2}<2 .
$$

This leads to $k_{\theta}<0.5 \mathrm{~cm}^{-1}$ and $k_{r}<0.9 \mathrm{~cm}^{-1}$ (for the plasma temperature $T_{e}=8 \mathrm{keV}$ ) to $2.8 \mathrm{~cm}^{-1}$ (for $T_{e}=$ $1 \mathrm{keV})$. Therefore, for the broadband turbulence, observed structures are expected to be limited to wavelengths $\lambda=2 \pi / k_{\theta} \geq 12 \mathrm{~cm}$, and thus the fluctuation level is likely to be underestimated. Whereas for MHD modes such as electron fishbones, or higher frequency TAEs, which typically have $k_{\theta} \leq 0.5 \mathrm{~cm}^{-1}$, the signal is only weakly attenuated.

\section{DIAGNOSTIC SETUP}

The heterodyne radiometer on the Tore Supra tokamak has been recently upgraded to have 32 measuring channels with $1 \mathrm{GHz}$ spacing and $500 \mathrm{MHz}$ bandwidth. ${ }^{16}$ It is used to measure the ECE in the first-harmonic ordinary $(\mathrm{O})$ mode $(\vec{E} \| \vec{B}, \vec{k} \perp \vec{B})$ over the frequency range 78 to $110 \mathrm{GHz}$ and in the second harmonic extraordinary (X) mode $(\vec{E} \perp \vec{B}, \vec{k} \perp \vec{B})$ over the frequency range 94 to 126.5 GHz. Fast acquisition for the profile radiometer is done with the video bandwidth $B_{V}=42 \mathrm{kHz}$ at a sampling rate of $84 \mathrm{kHz}$.

To perform measurements of the electron temperature fluctuations, one radiometer channel is split into two (Fig. 2) branches. The branch used for fluctuation measurements is again split into two. On each of these two subsequent branches, an intermediate frequency yttrium iron garnet (YIG) filter with narrow bandwidth is 


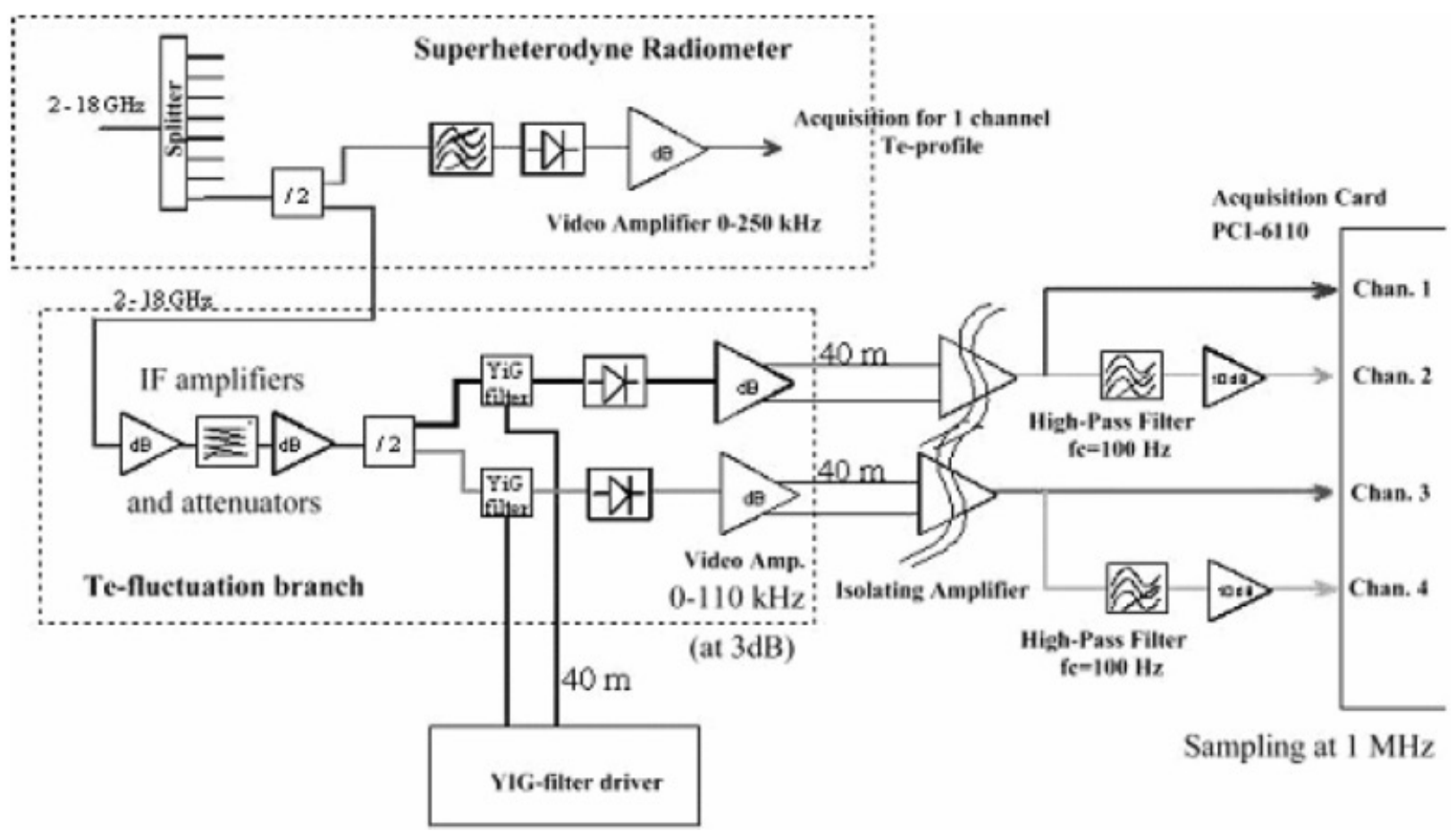

Fig. 2. Principal electronic scheme to measure electron temperature fluctuations on Tore Supra.

introduced. Its central frequency is remotely monitored by a driver between 6 and $18 \mathrm{GHz}$, allowing the observation volume in the plasma to be shifted radially. The filters (WJ5908 from Watkins-Johnson) have a -3-dB bandwidth of $110 \mathrm{MHz}$ at $6 \mathrm{GHz}$ and $118 \mathrm{MHz}$ at 17.6 GHz. The insertion loss of these filters is $3 \mathrm{~dB}$. After the intermediate frequency filters, a Schottky diode detector and a video amplifier, with a $-3-\mathrm{dB}$ bandwidth $B_{V}$ of about $110 \mathrm{kHz}$, are placed inside the isolation box. For each branch, two digitalized channels are acquired with a range of \pm 2048 bits: the unfiltered signal, labeled DC, and the high-pass-filtered, 10-dB-amplified, fluctuating part of the signal, labeled AC. This AC signal provides a larger useful range of signals with respect to the digitalization error.

It should be noted that signals of the four channels are oversampled at $1 \mathrm{MHz}$, which is beneficial for reducing aliasing effects. Conversely, the rather low video bandwidth implies that the high-frequency tail of the spectra $(>220 \mathrm{kHz})$ is unusable for the analysis of the spectral characteristics of "real" temperature fluctuations even with a correction for the gain of the video amplifiers and, for time lags smaller than $1 /\left(2 B_{V}\right)=$ $4.5 \times 10^{-6} \mathrm{~s}$, poor confidence must be given to the cross-correlation functions.

All measurements reported in this paper have been performed in the first-harmonic $\mathrm{O}$ mode. Depending on the toroidal magnetic field in the plasma center, a few measuring scenarios are possible for the correlation ECE on Tore Supra. For $B_{t}=3.4 \mathrm{~T}$, the radial region of observation is $-0.6<r / a<-0.2$, and for $B_{t}=4 \mathrm{~T}$, it is $-0.05<r / a<0.4$. The value of the Shafranov shift depends on the heating regime and typically varies between 5 and $12 \mathrm{~cm}$. The two probed volumes must have a radial extension that is much larger than their radial separation in order to fulfill the basic assumption of detecting radiation coming from the same plasma volume. Radial broadening of the ECE occurs due to relativistic effects and depends strongly on plasma temperature and more weakly on density. ${ }^{16}$ The full-width at half-maximum of the emission varies on Tore Supra between two extremes: $4 \mathrm{~mm}\left(T_{e}=1 \mathrm{keV}, n_{e}=2.5 \times 10^{19} \mathrm{~m}^{-3}\right)$ and $35 \mathrm{~mm}\left(T_{e}=8 \mathrm{keV}, n_{e}=2.5 \times 10^{19} \mathrm{~m}^{-3}\right)$. It has been found that the thermal noise is decorrelated if the separation of the two central frequencies (in terms of radii) is larger than the radial width of the filters of about 2.4 to $2.7 \mathrm{~mm}$ (central magnetic field $3.8 \mathrm{~T}, B_{\text {if }}=110$ to $118 \mathrm{MHz}$ at $-3 \mathrm{~dB}$ for different values of the filter central frequency). This has been verified by precise fine-tuning of the central frequency position of one filter on a shot-toshot basis (Fig. 3). Decorrelation is achieved for a radial separation larger than $3 \mathrm{~mm}$, as seen from the decay of the correlation coefficient. For the plasma with the strong additional heating, the two sample volumes are five to 10 times larger than they are radially shifted. However, for low-temperature plasmas (achieved at low plasma current with no additional heating), the situation is slightly different. 


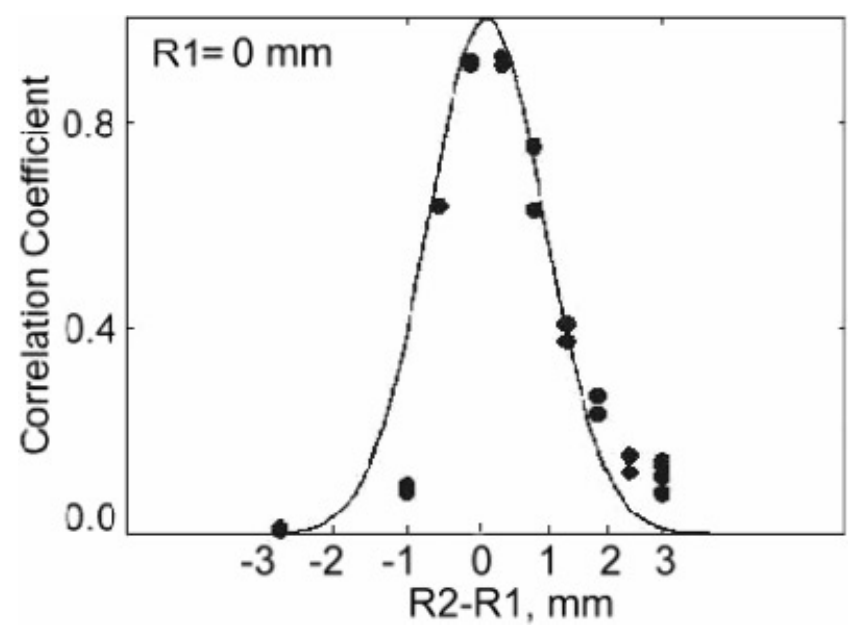

Fig. 3. Correlation coefficient of the ECE signal. The radial position of the first channel is fixed, while the second one shifts in the radial direction by fine-tuning of the central frequency of the YIG filter.

It is important to mention that the ripple in Tore Supra reaches $5.5 \%$ at the edge, leading to a mismatch between the iso- $\boldsymbol{B}$ lines, which determine the localization of the investigated volume, and the flux lines to which turbulence is perpendicular (Fig. 4). Consequently, for small distance between the two channels, the same field line would cross the two volumes. This could be impor- tant for the MHD mode localization, such as $m / n=3 / 1$ or $4 / 1$ on Tore Supra, and causes the same problem for the profile ECE radiometer. If the distance between two channels is smaller than a certain number, there would be a contribution of turbulent fluctuations originating from the same flux surface to both channels, making the turbulence propagation studies more complicated.

The minimum distance that can be reached without including this spurious effect is determined as

$$
\Delta R>w_{\mathrm{ECE}} \tan (\alpha) .
$$

The beam waist $w$ is equal to $6 \mathrm{~cm}$ and is taken to be the same for the two channels. The angle $\alpha$ is the angle between the curvature of the iso- $\boldsymbol{B}$ and the flux lines. The angle $\alpha$ can be written in the following form:

$$
\tan (\alpha)=N_{c} \sin \left(N_{c} \phi\right) \Delta B_{\varphi}
$$

where

$$
\begin{aligned}
N_{c}= & \text { number of toroidal field coils (18) } \\
\phi= & \text { ECE radiometer viewing angle with respect } \\
& \text { to port axis }(3.5 \mathrm{deg}) \\
\Delta B_{\varphi}= & \text { magnetic ripple that depends on radial posi- } \\
& \text { tion, varying between } 0.18 \% \text { (in the center) } \\
& \text { and } 0.93 \% \text { (at } r / a=+0.4, \text { low-field side) }
\end{aligned}
$$

This corresponds to $\alpha$ between 1.6 and $8.5 \mathrm{deg}$, respectively. Consequently, the spurious effect is avoided when

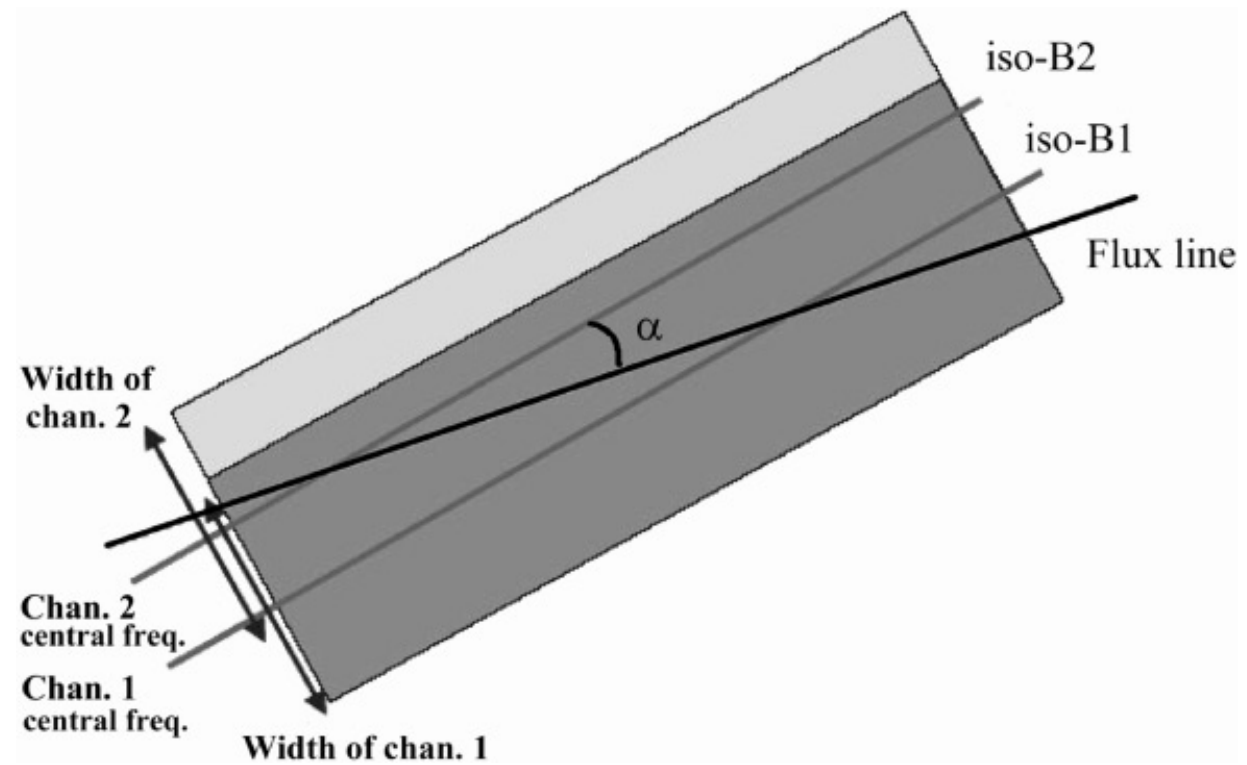

Fig. 4. Schematic view of the integration volume in the equatorial plane as well as two iso-B lines and one flux line. As the distance between the two decreases, the mixing of the information comes from not only the width of the beams but also from the difference between the iso-B and the flux lines. 
the radial separation is $\geq 1.5 \mathrm{~mm}$ in the plasma center and for distances $\geq 8 \mathrm{~mm}$ at $r / a=+0.4$.

\section{ESTIMATION OF THE MEASUREMENT ERROR}

\section{IV.A. Signal-to-Noise Ratio for the Tore Supra Radiometer}

ECE radiation coming from the plasma is attenuated by waveguide losses and millimetric attenuator for total attenuation $A=4 \mathrm{~dB}+17 \mathrm{~dB}=21 \mathrm{~dB}$. The $17-\mathrm{dB}$ attenuator is required for optimizing the signal level of the intermediate frequency components for the full temperature range (1 to $10 \mathrm{keV}$ ) for the 32-channel profile ECE radiometer. ${ }^{16}$ The source temperature $T$ seen by the radiometer can be defined as follows:

$$
T=\frac{T_{e}}{A}+T_{R N}+T_{E M},
$$

where

$$
A=126 \text { (for 21-dB attenuation) }
$$

$T_{R N}=$ equivalent radiometer temperature noise

$T_{E M}=$ parasitic noise due to electromagnetic pickup.

Following Ref. 17, the noise equivalent power (NEP) for one polarization direction due to fluctuations of the intensity of the thermal radiation can be written as

$$
\mathrm{NEP}=k T\left(B_{\mathrm{if}} 2 B_{V}\right)^{1 / 2},
$$

where $B_{\text {if }}$ and $B_{V}$ are the intermediate frequency and video bandwidths, respectively. If one assumes $T_{R N}$ to be about $10000 \mathrm{~K}$ (due to the mixer and the first intermediate frequency amplifier; the Schottky detector noise is negligible in case of strong intermediate frequency amplification), the minimum detectable temperature difference $\widetilde{T}_{\text {min }}$ is obtained when the blackbody radiated power is equal to the NEP (i.e., when the signal-to-noise ratio is unity):

$$
k \frac{\widetilde{T}_{\min }}{A} B_{\mathrm{if}}=k\left(\frac{T_{e}}{A}+T_{R N}+T_{E M}\right)\left(B_{\mathrm{if}} 2 B_{V}\right)^{1 / 2},
$$

which can be rewritten as

$$
\frac{\widetilde{T}_{\min }}{\bar{T}_{e}}=\sqrt{\frac{2 B_{V}}{B_{\mathrm{if}}}}\left(1+\frac{T_{R N} A}{\bar{T}_{e}}+\frac{T_{E M} A}{\bar{T}_{e}}\right) .
$$

If $T_{e} / A \gg T_{R N}, T_{E M}$, Eq. (12a) reduces to

$$
\frac{\widetilde{T}_{\min }}{\bar{T}_{e}}=\sqrt{\frac{2 B_{V}}{B_{\text {if }}}} .
$$

For $B_{V}=110 \mathrm{kHz}$ and $B_{\text {if }}=110 \mathrm{MHz}$ (both values are taken at $-3 \mathrm{~dB}$ ), the minimum detectable normalized temperature is of $4.5 \%$ (the thermal noise level). In experiments, however, it is found that if the mean signal measured by the acquisition is smaller than 300 bits, the normalized rms $\widetilde{T}_{\min } / \bar{T}_{e}$ is highly influenced by the $\left(T_{R N}+T_{E M}\right)$ term and, thus, is dominated by parasitic noise. These results are further confirmed by analysis of the correlated noise in the next section. It is important to mention that if any modes with a narrow bandwidth are present in the plasma, Eq. (12c) will be modified to

$$
\frac{\Delta T_{\min }}{\bar{T}_{e}}=\sqrt{\frac{B_{N B M}}{B_{R}}},
$$

where

$$
\begin{aligned}
B_{N B M}= & \text { bandwidth of the mode }\left(0<B_{N B M}<B_{V}\right) \\
B_{R}= & \text { bandwidth corresponding to "effective" ra- } \\
& \text { dial structure size }\left(0<B_{R}<B_{\text {if }}\right) .
\end{aligned}
$$

The radial width $W_{\text {if }}$ is determined by the radial resolution of the diagnostic. In experiments reported in this paper, real plasma structures are much larger than the radial limit set up by the $B_{\text {if }}$ and the relativistic broadening, so $B_{R}=B_{\text {if }}$ in Eq. (12d). The confirmation of Eq. (12d) will be demonstrated in Sec. V.B.

It is important to realize that in a plasma with low optical depth, density fluctuations can also influence temperature fluctuation measurements. This is the case if the latter are performed near the plasma edge. However, in the analysis performed is this section, the plasma is assumed to be optically thick for the first harmonic $\mathrm{O}$ mode ECE (see Table I for examples); hence, one can neglect the influence of the density fluctuations on the ECE spectra.

TABLE I

Examples of the Optical Depth for the First Harmonic O Mode for Various Temperature and Density Values* on Tore Supra

\begin{tabular}{|l|l|c|c|}
\hline $\begin{array}{c}\text { Radial } \\
\text { position } \\
(r / a)\end{array}$ & $\begin{array}{c}T_{e} \\
(\mathrm{keV})\end{array}$ & $\begin{array}{c}n_{e} \\
\left(10^{19} \mathrm{~m}^{-3}\right)\end{array}$ & $\begin{array}{c}\text { Optical Depth } \\
\text { (First Harmonic, } \\
\text { O-Mode) }\end{array}$ \\
\hline 0 & 2.5 & 2 & 5.4 \\
0.4 & 2.1 & 1.65 & 4.3 \\
0 & 4 & 3 & 12.5 \\
0.4 & 3.35 & 2.5 & 10 \\
\hline
\end{tabular}

*Assuming parabolic temperature and density profiles. 
For the estimation of the statistical noise level in the case of cross correlation between two ECE channels, the following expression has often been used ${ }^{10,15}$ :

$$
\Delta\left[\frac{\widetilde{T}_{e, \mathrm{rms}}}{\bar{T}_{e}}\right] \cong \frac{1}{\sqrt[4]{M / C}} \sqrt{\frac{\sigma_{1}}{\bar{S}_{1}} \frac{\sigma_{2}}{\bar{S}_{2}}},
$$

where

$$
\begin{gathered}
\sigma_{1}, \sigma_{2}=\underset{\tilde{S}_{2}}{\text { standard deviations of fluctuations } \tilde{S}_{1} \text { and }} \\
M=\text { total number of samples } \\
C=\begin{array}{l}
\text { numerical factor for Tore Supra measure- } \\
\text { ments resulting from oversampling. }
\end{array}
\end{gathered}
$$

It is useful to remember that the "total" acquisition sampling is $1 \mathrm{MHz}$ for the ECE correlation setup; however, $2 B_{V}(-3 \mathrm{~dB})=220 \mathrm{kHz}$ (Refs. 18 and 19); thus, $C=$ 4.54. Error sequence estimation from a series of $15 \mathrm{ohmic}$ pulses on Tore Supra (Fig. 5, open circles) suggests that Eq. (13) overestimates somewhat the statistical noise level for ECE correlation measurements if $M \geq 2 \times 10^{6}$, while for smaller $M$ a satisfactory agreement is found. ${ }^{18}$ For example, to resolve a fluctuation amplitude of $0.1 \%$, it is necessary to have $M=3 \times 10^{6}$ samples to get an error level of $0.05 \%$.

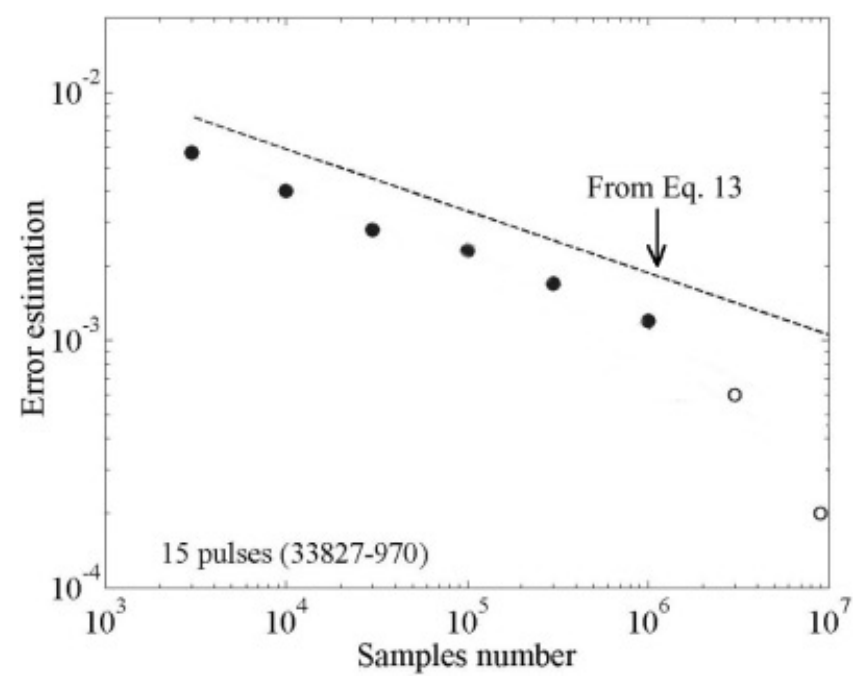

Fig. 5. An error estimation for $\widetilde{T}_{e} / \bar{T}_{e}$ measured by correlation ECE on Tore Supra. Closed and open circles are experimentally obtained results for low and high numbers of $M$, respectively. A comparison with the error estimation given by Eq. (13) is shown by a dashed line.

\section{IV.B. Correlated Noise}

From Eq. (5), the fluctuation level has been estimated for more than 180 plasma shots (366 sets of $3 \times$ $10^{6}$ samples) from the DC and AC signals by calculating the cross correlation of the normalized signals. When the radial channel separation exceeds the decorrelation length by several centimeters and the measured signal in bits is high enough to surpass the 300-bit limit, the value of $\max \left(R_{12}\right)$ has been taken instead of $R_{12}(0)$ in the interval $|\tau|<100 \mu$ s. This step is justified when the radial structure propagation in the plasma results in a $\max \left(R_{12}\right)$ value at the nonzero time lag. For the DC signals, $\max \left(R_{12}\right)$ cannot measure fluctuations below $0.3 \%$, because the fluctuating part of the signal is smaller than 1 bit for $\bar{T}_{e}^{\text {bits }}<300$ bits. The $10-\mathrm{dB}$ amplification of the AC signals provides a more reliable signal for lower values of $\bar{T}_{e}$. However, the gain is actually marginal (see Fig. 6b), as indicated by a correlated noise that is present between 80 and $120 \mathrm{kHz}$ (Fig. 7) at an amplitude of $0.7 \mathrm{~dB}$ (before amplification). This level of noise is almost the same as found from the rms $(\sim 0.6 \mathrm{~dB}$; see Fig. 6a), indicating that almost all the noise is correlated. It has been found that this noise originates from the electromagnetic environment in Tore Supra and comes from the low-frequency section. To avoid this parasitic pickup, 40-m cables between video amplifiers and differential amplifiers will be substituted by optical cables. For the data shown in Fig. 6a, signals were bandpass filtered between 20 and $150 \mathrm{kHz}$. Thus, the dashed line is the best fit of these data with Eq. (12b) but taking $B_{V}^{\prime}=B_{V}(-3 \mathrm{~dB})-20$ $\mathrm{kHz}=90 \mathrm{kHz}$, instead of the full video bandwidth $B_{V}$ $(-3 \mathrm{~dB})=110 \mathrm{kHz}$. The scatter in the 366 sets of measurements is very low: More than $90 \%$ of the data lie between 0.15 and $0.30 \%$ when $\bar{T}_{e}^{\text {bits }}$ exceeds 300 bits. Indeed, almost all shots with $\max \left(R_{12}\right)^{1 / 2}>0.35 \%$ exhibit high-frequency modes that, as will be discussed in Sec. V, are identified, for the most part, as TAE modes.

To estimate the frequency dependence of the noise, the cross-correlation spectrum of the two signals for a shot with a low fluctuation level $(\sim 0.20 \%)$ has been compared to the one without plasma. For the latter case, the cross-spectral density has been adjusted by multiplying by a constant, chosen so as to obtain the same spectrum for frequencies above $250 \mathrm{kHz}$. This is fully justified if we assume that the thermal noise is uncorrelated. From the comparison between spectra with and without the plasma (Fig. 7), it has been concluded that details of the spectra above $300 \mathrm{kHz}$ are seen to be identical, and the large increase of the signal below $220 \mathrm{kHz}$ can still be assessed. In this case, however, a correction for a gain of the video amplifier as a function of a frequency is needed for the proper estimation of the fluctuation amplitude. One can notice that the parasitic noise at $100 \mathrm{kHz}$ is just above the signal level with plasma, whereas the signal 


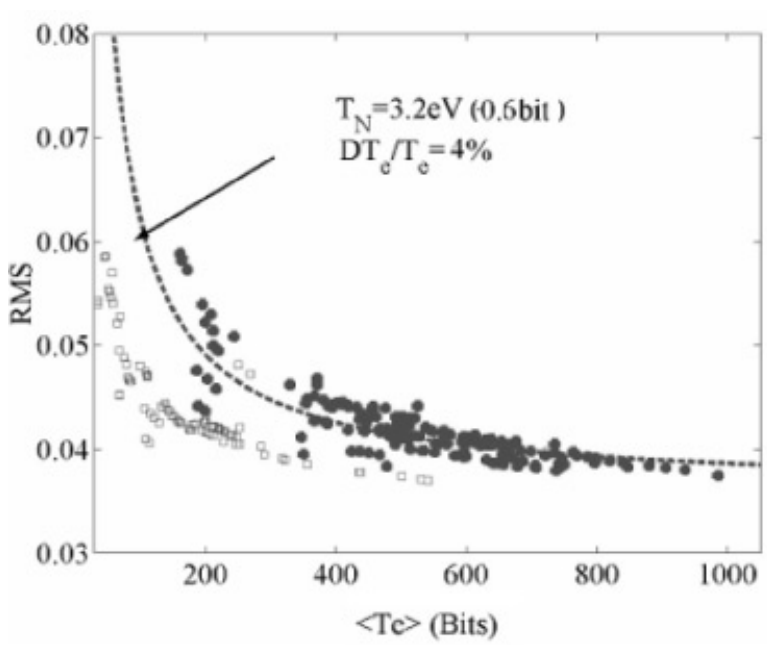

(a)

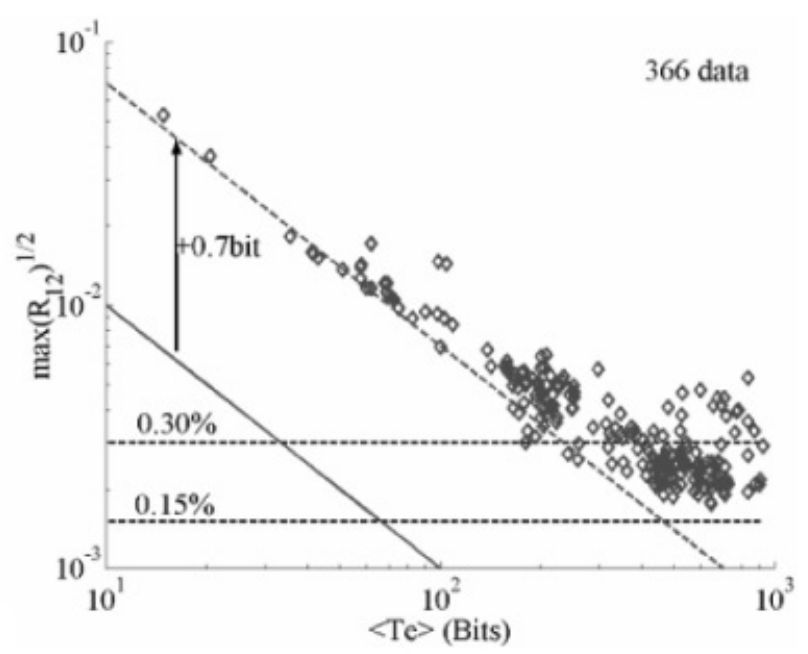

(b)

Fig. 6. (a) RMS of the normalized signal as a function of the time-averaged signal. Closed circles indicate cases for which the radiometer sensitivity is high $(>110$ bits $/ \mathrm{keV})$. The dashed line is the best fit of these data with Eq. (12b) but for the 20 to $150 \mathrm{kHz}$ bandpass filtered data. (b) Square root of the cross correlation as a function of the average temperature measured at its maximum value for the AC signal. Signals have been filtered with a numerical 20 to $150 \mathrm{kHz}$ bandpass filter (to suppress low-frequency MHD effects and the meaningless high-frequency tail). For the signal with $\bar{T}_{e}^{\text {bits }}<300$ bits, the $\max \left(R_{12}\right)$ is dominated by the 0.7 -bit parasitic noise indicated by the slanting dashed line. The slanting solid line indicates the estimated noise level if the parasitic electromagnetic noise were absent. For values of $\bar{T}_{e}^{\text {bits }}>300$ bits, useful information on the amplitude of plasma structures can be obtained. Horizontal dashed lines indicate the two fluctuation amplitude levels mentioned in the text.

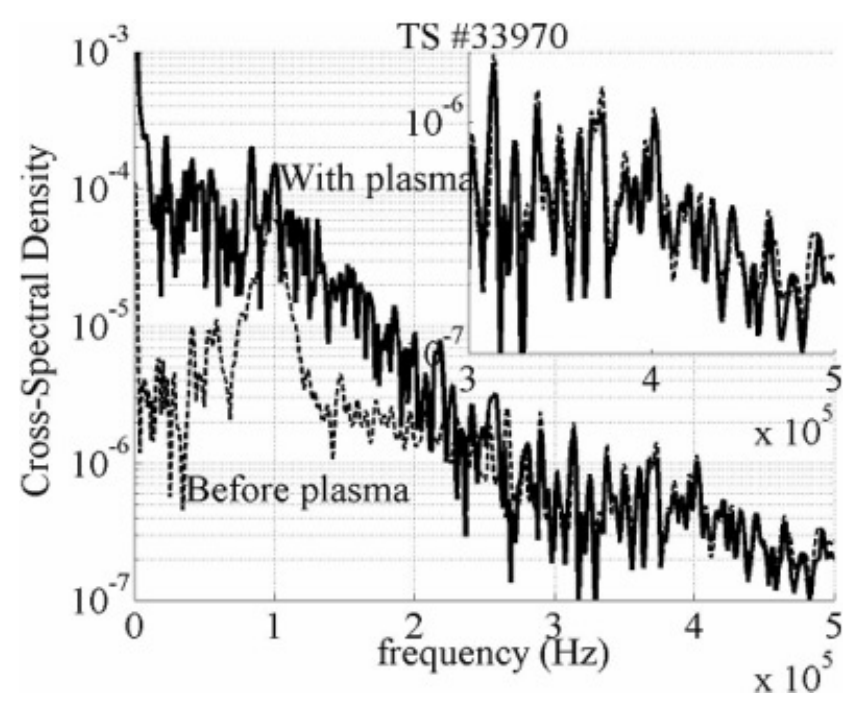

Fig. 7. Cross-spectral density of the signals measured before the $\operatorname{shot}(t=-5$ to $-2 \mathrm{~s})$ and with plasma ( $t=4$ to $7 \mathrm{~s})$. The fluctuation level over the 20 to $150 \mathrm{kHz}$ frequency range is about $0.2 \%$.

with plasma is not distorted, indicating that the noise is not underestimated. We therefore infer that the level $(0.15$ to $0.30 \%$ ) for the spectrum obtained from the measurement with the plasma is significantly above the noise.

\section{FIRST EXPERIMENTAL RESULTS WITH RF HEATING}

Correlation ECE measurements performed on other machines (RTP, TEXT-U, DIII-D, Alcator C-Mod) have shown that the broadband turbulence level is usually on the order of 0.5 to $3 \%$ in amplitude with $k_{\theta}=1$ to $3 \mathrm{~cm}^{-1}$ for the poloidal wavenumber. ${ }^{3,6,15,20}$ On Tore Supra, despite the ability of the diagnostic to go as low as $0.15 \%$ for the minimum detectable temperature fluctuation amplitude for the broadband fluctuations, these wave numbers are highly attenuated in amplitude and downshifted in frequency (see Sec. II) because of the large poloidal beam waist ( $\sim 6 \mathrm{~cm}$ in the plasma center). Nevertheless, the diagnostic proved to be very useful in studies of highfrequency narrowband structures with the amplitude smaller than $1 \%$. The main results are presented in the next two sections. A special case for the broader bandwidth fluctuations during strong rf heating is discussed in the last section.

\section{V.A. Electron Fishbone Mode in Lower Hybrid Current Drive Plasmas}

A fast $(7.7-\mathrm{kHz})$ MHD mode has been observed near the minimum of the safety factor $q(r)$ in plasmas with fully noninductive lower hybrid current drive (LHCD) (Fig. 8). This mode can be superimposed on a slower 


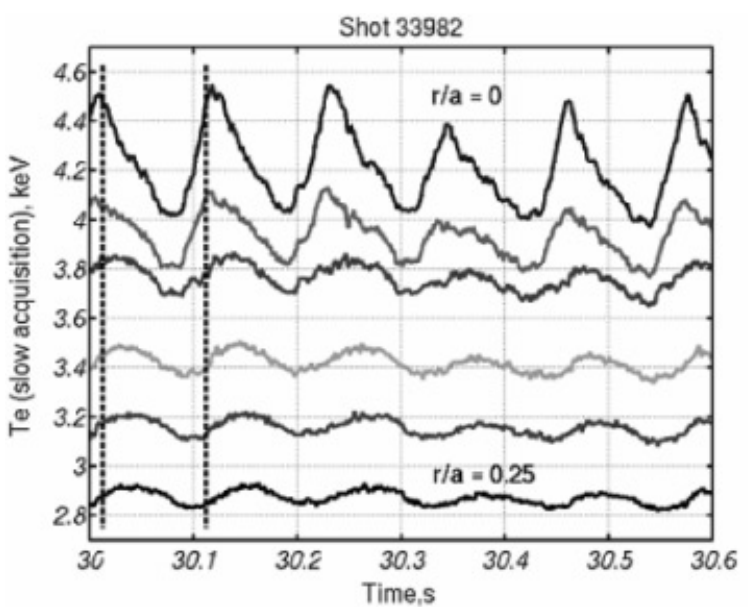

(a)

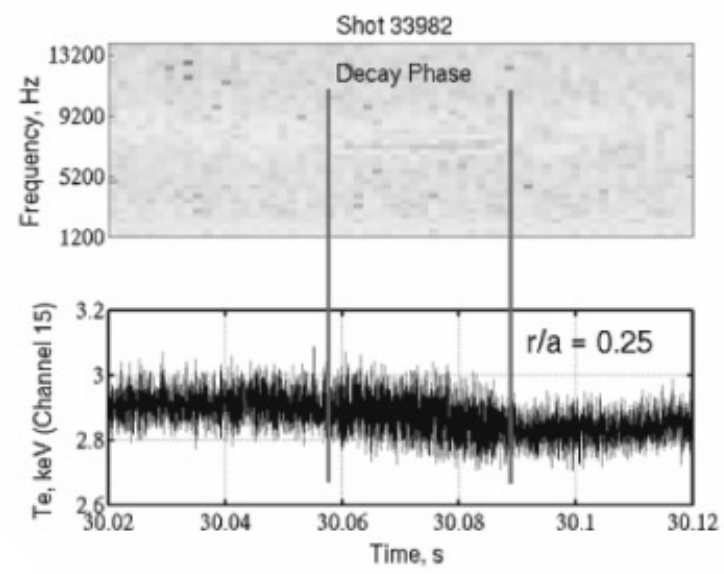

(b)

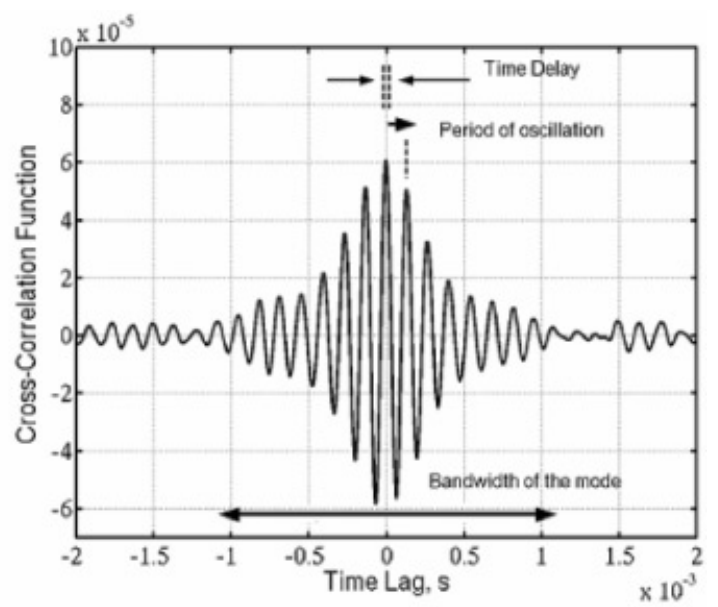

(c)

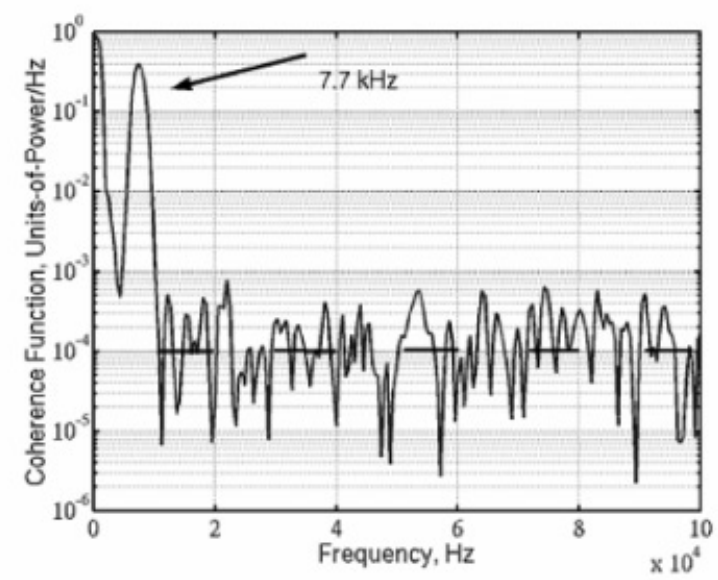

(d)

Fig. 8. (a) Observation of the electron fishbone mode by the profile radiometer during the phase with $T_{e}$ oscillations. (b) Spectrogram for the ECE channel at $r / a=0.25$. (c) From the correlation ECE, after 5-kHz high-pass filtering, the relative amplitude of the fast mode, as well as the bandwidth, can be determined. The minor time delay at zero lag is smaller than $1 / 2 B_{V}$ and, thus, an artifact of the calculation. (d) The cross-coherence function gives the frequency of the mode. The dashed line gives the statistical noise level estimation.

double-tearing $m / n=2 / 1$ or $3 / 2$ mode and/or oscillations of the central electron temperature. ${ }^{21}$ Unlike the large 2/1 perturbation, this fast mode is barely detectable by Mirnov coils. As follows from the MHD analysis, it cannot be interpreted as a conventional tearing mode. This instability is likely to be the so-called "electron fishbone" mode observed earlier on DIII-D, FTU, and HL1-M in LHCD and/or ECRH plasmas. ${ }^{22-26}$ On Tore Supra, however, it would correspond to the destabilization of the $m / n=2 / 1$ resistive double-kink mode by barely trapped fast electrons with the energy above $30 \mathrm{keV}$. The electron fishbone mode can be used as a test bed for the correlation ECE diagnostic. Following Eq. (5), the relative amplitude $\widetilde{T}_{e} / T_{e}$ averaged over $3 \mathrm{~s}$ of sampling is estimated to be about $0.8 \%$, as calculated from the zero time lag of the cross-correlation function (Fig. 8c). This is well above the statistical noise level. The mode amplitude may vary depending on the phase of an oscillation, having $\widetilde{T}_{e} / T_{e} \approx 1 \%$ during the decay of oscillation and less than $0.4 \%$ during its rise. The bandwidth of the mode is equal to $450 \mathrm{~Hz}$, as determined from the envelope shown in Fig. 8c. From the profile radiometer ${ }^{16}$ measurements, the radial structure size $\lambda$ is estimated to be equal to 8 to $10 \mathrm{~cm}$, meaning that $k_{r}=2 \pi / \lambda=0.8$ to $0.6 \mathrm{~cm}^{-1}$. This value is smaller than the upper limit set up by Eq. (8a), and thus the estimation of the mode amplitude is justified. With the radial separation of $4.6 \mathrm{~cm}$ between two correlation ECE channels and the time delay of $-1.4 \times 10^{-5} \mathrm{~s}$, the radial propagation velocity of 3.3 $\mathrm{km} / \mathrm{s}$ toward the plasma center has been deduced. More 
information on the electron fishbone mode observation in Tore Supra can be found elsewhere..$^{24,25}$

\section{V.B. Observation of Hot Ion Instabilities During ICRH}

Two branches of high-frequency (40 to 60 and 90 to $150 \mathrm{kHz}$ ) modes have been observed for the first time on Tore Supra by correlation ECE in ICRH discharges with a fraction of LHCD $\left[P_{\mathrm{ICRH}}=3\right.$ to $7 \mathrm{MW}$, $P_{\mathrm{LH}}=1.7$ to $3 \mathrm{MW}, I_{p} \leq 0.6 \mathrm{MA}, n_{e}(0)=3$ to $5 \times$ $\left.10^{19} \mathrm{~m}^{-3}, \mathrm{H}(\mathrm{D})\right]$. Measurements have been made at $r / a=0.12$ to 0.2 on the LFS (that is, in the temperature gradient region). The channel separation was about $5 \mathrm{~mm}$, meaning that signals are received from highly overlapping plasma sample volumes but still are sufficiently separated to decorrelate the thermal noise. A typical cross-spectral density with two clearly distinguishable frequencies at 48.8 and $142.5 \mathrm{kHz}$ is shown in Fig. 9a. The corresponding relative amplitudes are 0.28 and $0.33 \%$ for the $48.8-$ and $142.5-\mathrm{kHz}$ modes, respectively, as calculated from the biased cross-correlation function at the zero time lag. The data are bandpass filtered (with the filter depending on the frequency of interest) and corrected for the gain of the video amplifier. Because the separation between two channels is small, the radial propagation velocity cannot be deduced from the measurement. From cross-correlation functions, and considering Eq. (7), bandwidths are determined to be 2.5 $\mathrm{kHz}$ for the $48.8 \mathrm{-kHz}$ mode and $0.5 \mathrm{kHz}$ for the 142.5 mode. Applying Eq. (12d) for the $142.5-$ and $48.8-\mathrm{kHz}$ modes, the minimum detectable temperature fluctuations $\widetilde{T}_{\min } / \bar{T}_{e}$ for a single channel are calculated to be 0.21 and $0.42 \%$, respectively, in agreement with observations. Therefore, a cross-correlation analysis is absolutely required to reveal the amplitude of the $48.8-\mathrm{kHz}$ mode, and thus mode amplitude measurements reported in this section are justified.

Modes of such high frequency $(>40 \mathrm{kHz})$ cannot themselves be ascribed to any "classical" MHD activity, such as tearing or kinklike activity; however, they can coexist with $2 / 1$ or $3 / 2$ double-tearing and electron fishbone modes. Due to the LHCD and the low loop voltage $(<100 \mathrm{mV})$, most plasmas with high-frequency modes have a slightly reversed central magnetic shear. It is known that ICRH-produced (or neutral beam injection) energetic (minority) ions of hydrogen can destabilize the so-called TAEs (Refs. 27 and 28) in a plasma with deuterium majority ions. The radial position of the measurement, $r / a=0.12(R=2.57 \mathrm{~m})$, corresponds to the approximate location of minimum safety factor $q_{\min }$. The $q_{\min }$ has a value between 1.2 and 2 depending on the particular plasma conditions, evidenced by the localization of $2 / 1$ or $3 / 2$ double-tearing and/or electron fishbone modes with their typical frequencies of 1 and $8 \mathrm{kHz}$ (Ref. 25), respectively, or calculated by means of the one-dimensional transport code CRONOS (Ref. 29). The high-frequency instability is observed between $r / a=-0.6$ on the high field side and $r / a=$ 0.1 in the plasma center using the fluctuation reflectometer ${ }^{30}$ For the Tore Supra discharges discussed here, the Alfvén velocity $v_{A}$ is in the range between $0.6 \times 10^{7}$ and $1.0 \times 10^{7} \mathrm{~m} / \mathrm{s}$, which corresponds to the TAE frequency of 95 to $180 \mathrm{kHz}$ (Ref. 31). This frequency range is very close to the frequency of the mode measured in the plasma with $P_{\mathrm{ICRH}}=3.17 \mathrm{MW}, P_{\mathrm{LH}}=$ $2.6 \mathrm{MW}$, and $n_{e}=3.6 \times 10^{19} \mathrm{~m}^{-3}$ (see Fig. 9d). For those shots, the existence of the TAE gap for the modes with the toroidal $n \leq 10$ has been predicted by the code MISHKA (Refs. 32 and 33). The frequency of the fast mode depends on local density and injected ICRH power. The amplitude of fluctuations decays when the ratio between ICRH power and the electron density (Fig. 9c) increases; however, more shots with TAEs are required to obtain good statistics. For the discharge mentioned above, the energy of hot ions responsible for the excitation of TAEs is estimated to be above $250 \mathrm{keV}$ (Ref. 34). For densities higher than $5.5 \times 10^{19} \mathrm{~m}^{-3}$, no TAEs are excited.

In reversed shear plasmas on Tore Supra, the frequency of the TAEs follows the change in frequency of the slower MHD modes, such as 2/1 double-tearing or electron fishbone modes, or may depend on the phase of the oscillations of the central electron temperature. In shot \#34176, the TAE frequency increases while at the same time the density decays between 11 and $14 \mathrm{~s}$ (Fig. 9d). At 12.3 and $13.3 \mathrm{~s}$, the TAE frequency is oscillating in the counterphase, with respect to the oscillations of the electron temperature in the plasma center. ${ }^{31,35}$ Because the evolution of the temperature in the oscillation $(\mathrm{O})$ regime is thought to be linked to the development of the current density profile, ${ }^{21}$ this observation is a strong indication that the value of the safety factor at $r / a=0.1$ to 0.3 indeed evolves periodically during the $T_{e}$ oscillation cycle. Because the $q_{\mathrm{TAE}}$ traces out the density change during the $q$ profile oscillation, the value of the $v_{A}$ changes, thus altering the mode frequency. As evidenced by the density profiles obtained by means of the interferometer, the $m=(2+3) / n=2$ TAE in shot \#34176 may trace out $\sim 5$ to $6 \%$ change in density during the temperature oscillation cycle if $\Delta r / a\left(q_{\mathrm{TAE}}\right)= \pm 0.1$. The detailed analysis of the TAE frequency evolution in plasmas with oscillations of the central electron temperature can be found in Ref. 31.

The second, lower, frequency range (40 to $60 \mathrm{kHz})$ in which modes are observed lies outside the possible TAE range. These modes are also seen by the fluctuation reflectometer. They may correspond to geodesic acoustic modes ${ }^{31,36}$ (GAMs); however, their relation to the beta-induced Alfvén eigenmodes ${ }^{34}$ (BAEs) cannot be excluded, as the BAE gap is predicted by MISHKA calculations if the plasma compressibility is added. On Tore Supra, these modes could be excited by the energetic ions in ICRH discharges and have frequencies less 


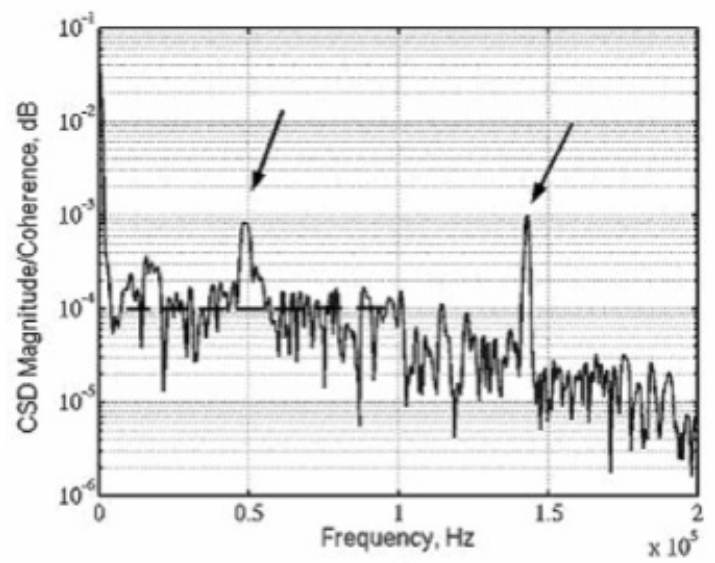

(a)

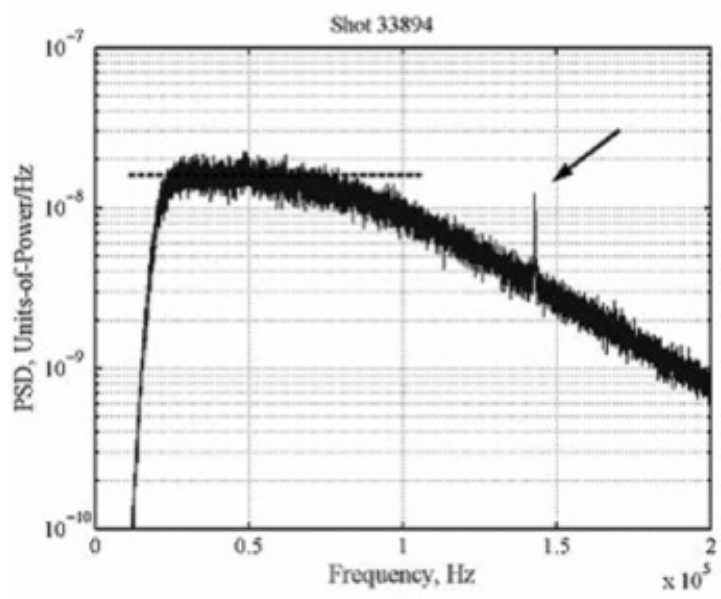

(b)

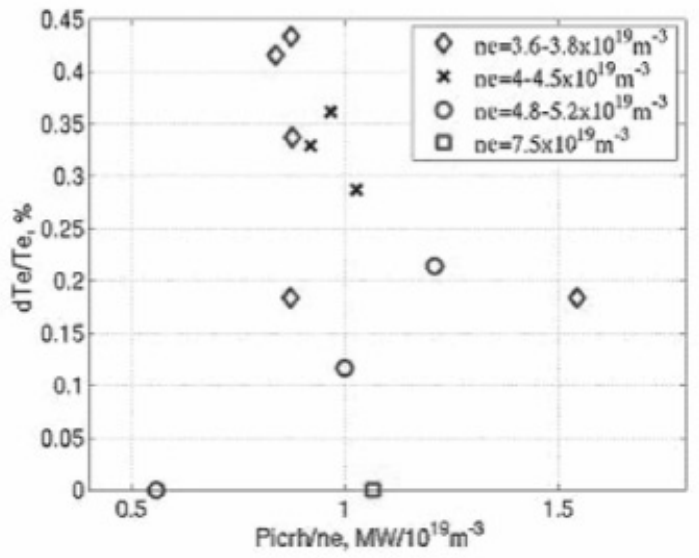

(c)

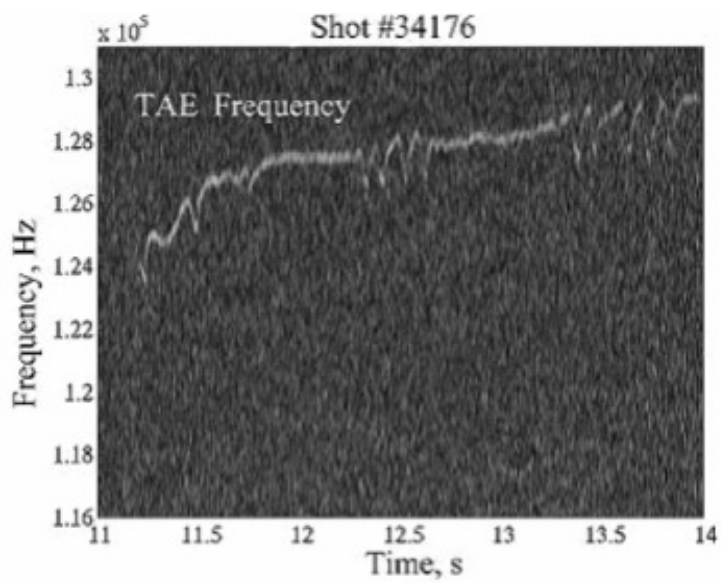

(d)

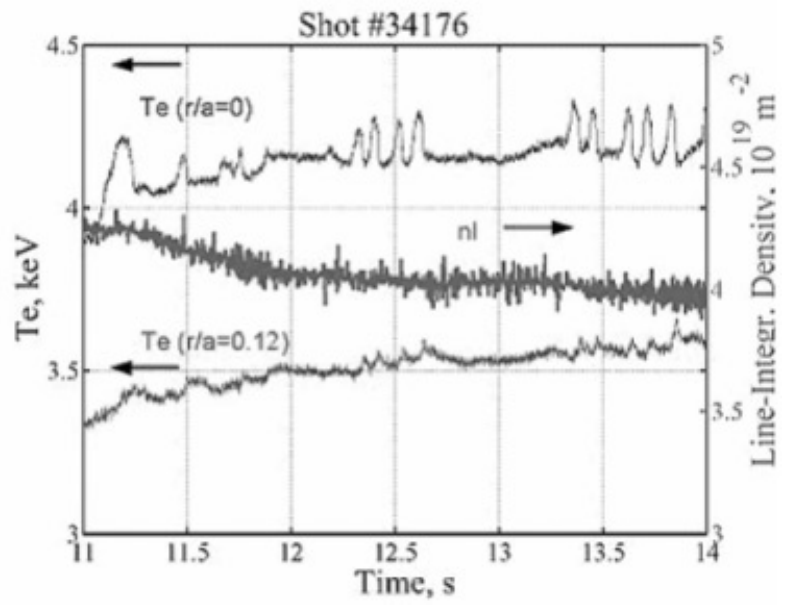

(e)
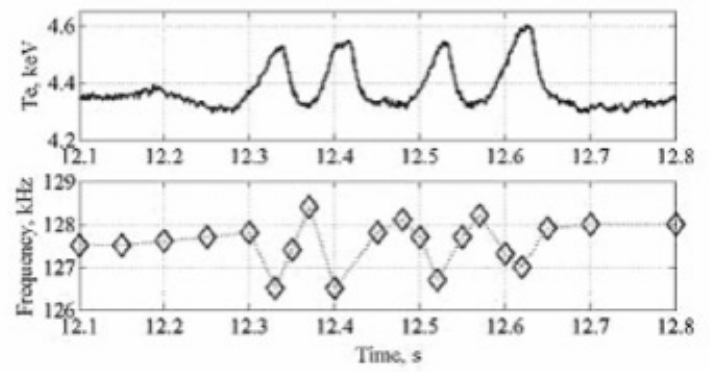

(f)

Fig. 9. (a) Cross-spectral power density shows a presence of high-frequency modes in combined LHCD and ICRH discharge \#33894. The statistical noise level is shown by a dashed line up to $100 \mathrm{kHz}$; for higher frequencies, overall spectra decay occurs due to the video filter finite bandwidth. (b) The autocorrelation spectrum for a 20-kHz high-pass-filtered single ECE channel shows a presence of the $142.5-\mathrm{kHz}$ mode, while the $48.8-\mathrm{kHz}$ mode is almost buried in the noise (dashed line is for the statistical noise level). (c) High-frequency-mode amplitudes for different values of the electron density as a function of the ICRH power shows a $P_{\mathrm{ICRH}} / n_{e}$ dependence of the TAE amplitude. (d) An overall TAE frequency increase from 124 to $129 \mathrm{kHz}$ in shot \#34176 (white trace) is observed during the general electron density decay (e). (f) The frequency of the TAE in the reversed-shear plasma for the same shot follows the dynamic change of the phase of lower-frequency temperature oscillations. 
than those of the TAEs. A detailed analysis with new experimental investigations will be performed in future campaigns.

\section{V.C. Broadband Fluctuations During the Strong rf Heating}

Although experiments were carried out with a rather large range of additional heating ( 0 to $9 \mathrm{MW}$, mainly from ICRH and $\mathrm{LH}$ ), temperature fluctuations vary very little in the core of the plasma $(|r / a| \leq 0.5)$. As has been mentioned in Sec. IV.B, the broadband (20- to 200-kHz) fluctuation amplitude is expected to be bounded between 0.15 and $0.30 \%$. As was mentioned in the beginning of the Sec. V, the diagnostic in its present state has a poor sensitivity for low- and medium-scale turbulence due to the strong spectral attenuation for $k_{\theta}>0.5 \mathrm{~cm}^{-1}$. Nevertheless, some interesting results have been observed in shots with additional high-power rf heating, compared to ohmic plasmas or plasmas with the lower additional power.

For the highest $\mathrm{rf}$ power (6.9 MW of ICRH and 2.1 MW of LHCD, $\left.n_{e}=7.5 \times 10^{19} \mathrm{~m}^{-3}\right)$, the fluctuation level is estimated to be about $0.48 \%$. The measurement has been performed in or close to the gradient zone at $r / a=0.15$ at the LFS. The temperature gradient of $d T_{e} / d R=11 \mathrm{keV} / \mathrm{m}$ corresponds to a dimensionless logarithmic gradient $\left|R_{0} d \log T_{e} / d R\right|=8$ (Fig. 10a). When compared to the prelude phase performed at low density $\left(n_{e}=3 \times 10^{19} \mathrm{~m}^{-3}\right)$ and low additional power $\left(P_{\mathrm{LHCD}}=1.6 \mathrm{MW}\right)$, for which the temperature gradient $d T_{e} / d R=5.9 \mathrm{keV} / \mathrm{m}$ (and thus $\left|R_{0} d \log T_{e} / d R\right|=7$ ), the temperature fluctuation amplitude is reduced by a factor of 2 for the frequency range between 20 and 100 $\mathrm{kHz}$ (Fig. 10b). The detailed analysis is under way.

\section{CONCLUSION}

Successful measurements of electron temperature fluctuations with the amplitude as low as $0.15 \%$ have been performed by means of radial correlation ECE diagnostic on Tore Supra, despite the fact that the poloidal resolution of the diagnostic is limited. The plasma core could be probed over a range of normalized radius $(-0.5<$ $r / a<0.4)$. For the first time on Tore Supra, the highfrequency modes have been observed by ECE in ICRH plasmas with LHCD fraction. These modes have been identified as TAEs (higher frequency branch) and, possibly, GAMs or BAEs (lower frequency branch), thus yielding information on the energy of the fast ion tail and the evolution of the safety factor.

In upcoming experimental campaigns, an emphasis will be given to detailed studies of the broadband turbulence and narrowband modes at various radial positions under different plasma conditions. Therefore, an improved correlation ECE diagnostic with a smaller sample volume and suppressed electromagnetic pickup is strongly

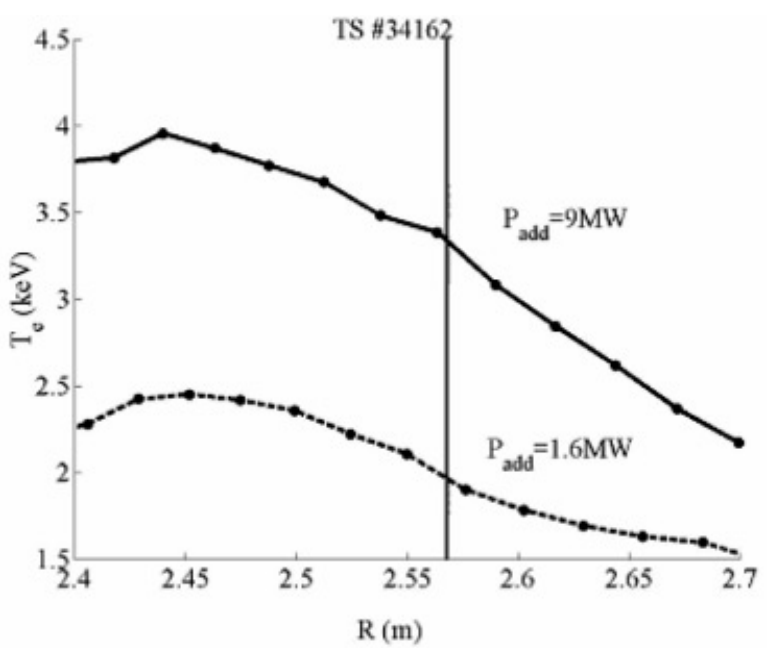

(a)

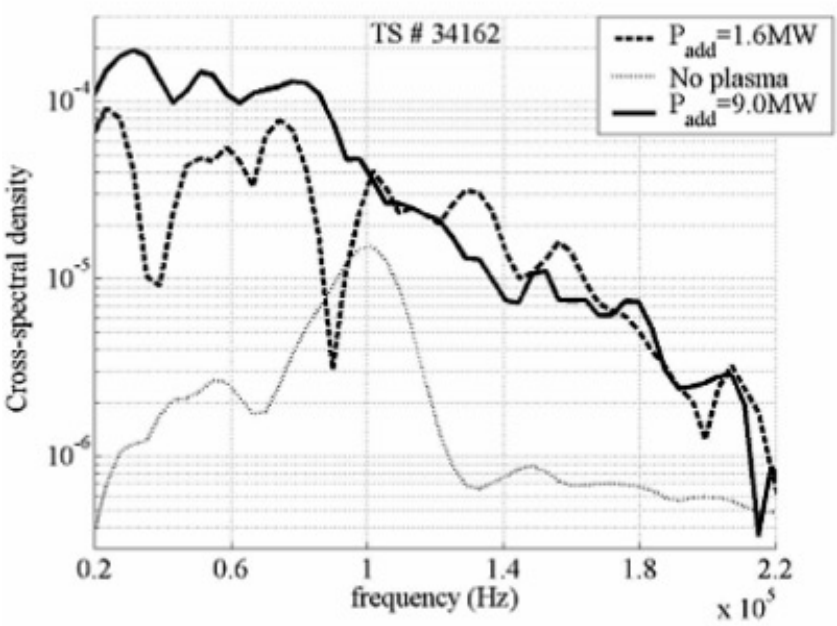

(b)

Fig. 10. (a) Electron temperature profiles and (b) crossspectral densities are measured at the same radial position in the same shot but in different time windows with different heating powers. The vertical line in (a) indicates the position of the correlation ECE measurement. The thin dashed line in (b) shows the cross spectrum without the plasma, and the thick dashed line represents the statistical noise level for the spectra with the plasma. Before fast Fourier transform processing, all signals were bandpass filtered between 20 and $200 \mathrm{kHz}$.

needed for the proper identification of the broadband turbulence on Tore Supra.

\section{ACKNOWLEDGMENTS}

The authors are thankful to G. Y. Antar (University of California at San Diego) and W. A. Peebles (University of California at Los Angeles) for providing YIG filters and the 
acquisition card, as well as for a stimulating discussion on properties of the thermal noise in ECE radiometry, and to T. Goodman (CRPP/SB/EPFL, Switzerland) for editing the manuscript.

\section{REFERENCES}

1. W. HORTON, "Drift Wave Turbulence and Anomalous Transport," Basic Plasma Physics, Vol. 2, p. 383, A. A. GALEEV and R. N. SUDAN, Eds., Elsevier, New York (1984).

2. G. CIMA et al., Phys. Plasmas, 2, 720 (1995).

3. C. WATTS, R. F. GANDY, T. D. REMPEL, and G. CIMA, Rev. Sci. Instrum., 66, 451 (1995).

4. S. SATTLER and H. J. HARTFUSS, Plasma Phys. Control. Fusion, 35, 1285 (1993).

5. S. SATTLER and H. J. HARTFUSS, Phys. Rev. Lett., 72, 653 (1994).

6. H. J. HARTFUSS et al., Plasma Phys. Control. Fusion, 38, A227 (1996).

7. B. H. DENG et al., Rev. Sci. Instrum., 72, 368 (2001).

8. V. S. UDINTSEV et al., Rev. Sci. Instrum., 72, 359 (2001).

9. C. WATTS et al., Nucl. Fusion, 44, 987 (2004).

10. C. WATTS et al., Rev. Sci. Instrum., 75, 3177 (2004).

11. D. VAN HOUTTE et al., Nucl. Fusion, 44, L11 (2004).

12. C. P. RITZ et al., Rev. Sci. Instrum., 59, 1739 (1988).

13. J. S. BENDAT and A. G. PIERSOL, Random Data: Analysis and Measurement Procedures, Wiley, New York (1986).

14. R. V. BRAVENEC and A. J. WOOTTON, Rev. Sci. Instrum., 66, 802 (1995).

15. B. H. DENG, PhD Dissertation, University of California at Davis (1999).

16. J. L. SEGUI et al., Rev. Sci. Instrum., 76, 123501 (2005).

17. H. J. HARTFUSS, "Coherent Versus Incoherent Detection Schemes," Proc. EC-7 Workshop, Hefei, China, May 9-11, 1989, p. 267 (1989).

18. V. S. UDINTSEV et al., "First Measurements of Electron Temperature Fluctuations by Correlation ECE on Tore Supra," Proc. 12th Int. Congress on Plasma Physics (ICPP), Institute of Physics, Bristol, United Kingdom (2004); available on the Internet at $\langle$ http://hal.ccsd.cnrs.fr/ccsd-00001778/en/ $\rangle$.
19. M. GONICHE, V. S. UDINTSEV, and J. L. SÉGUI, "Identification of Fast Particle Triggered Modes by Means of Correlation ECE in Tore Supra," Fusion Sci. Technol. (submitted for publication).

20. C. L. RETTIG, W. A. PEEBLES, J. LOHR, and M. E. AUSTIN, Rev. Sci. Instrum., 68, 484 (1997).

21. G. GIRUZZI et al., Phys. Rev. Lett., 91, 135001 (2003).

22. K. L. WONG et al., Phys. Rev. Lett., 85, 996 (2000).

23. F. ZONCA et al., "Resonant Excitation of Electron Fishbones in FTU and HL-1M," presented at 8th Easter Plasma Meeting on Reconnection and Turbulence in Magnetically Confined Plasmas, Turin, Italy, April 23-25, 2003.

24. P. MAGET et al., "First Observation of Electron Fishbones Associated to the Double-Kink Mode in Tore Supra," Proc. 32nd EPS Conf. Plasma Physics, European Conf. Abstracts, Vol. 29C, O2-002, European Physical Society (2005).

25. P. MAGET et al., "Temperature Oscillating Regimes in Tore Supra Diagnosed by MHD Activity," Nucl. Fusion, 46, 797 (2006).

26. F. IMBEAUX et al., Phys. Rev. Lett., 96, 045004 (2006).

27. D. BORBA et al., Plasma Phys. Control. Fusion, 46, 809 (2004).

28. O. ZIMMERMANN et al., "Excitation of Alfvén-Like Modes by Large 2/1 Tearing Modes on TEXTOR," Proc. 32nd EPS Conf. Plasma Physics, European Conf. Abstracts, Vol. 29C, P2-059, European Physical Society (2005).

29. V. BASIUK et al., Nucl. Fusion, 43, 822 (2003).

30. R. SABOT et al., "Density Fluctuation Studies in ToreSupra Core with X-Mode Reflectometry," Proc. 31 st EPS Conf. Plasma Physics, European Conf. Abstracts, Vol. 28G, P4.112, European Physical Society (2004).

31. V. S. UDINTSEV et al., Plasma Phys. Control. Fusion, $\mathbf{4 8}$, L33 (2006).

32. G. T. A. HUYSMANS et al., Phys. Fluids, B3, 1506 (1992).

33. G. T. A. HUYSMANS et al., Phys. Fluids, B5, 1545 (1993).

34. V. BASIUK et al., Nucl. Fusion, 44, 181 (2004).

35. V. S. UDINTSEV et al., "Electron Temperature Fluctuation Studies in Different Confinement Regimes by Means of Correlation ECE on Tore Supra," Proc. 32nd EPS Conf. Plasma Physics, European Conf. Abstracts, Vol. 29C, P1-037, European Physical Society (2005).

36. G. R. McKEE et al., Plasma Phys. Control. Fusion, 45, A477 (2003). 